\title{
A Novel Thermodynamic Model and Temperature Control Method of Laser Soldering Systems
}

\author{
Zhihua Chen, ${ }^{1}$ Cheng He, ${ }^{1}$ Ying Zheng, ${ }^{1}$ Xiaolong Shi, ${ }^{1}$ and Tao Song ${ }^{2,3}$ \\ ${ }^{1}$ Key Laboratory of Image Information Processing and Intelligent Control, School of Automation, \\ Huazhong University of Science and Technology, Wuhan, Hubei 430074, China \\ ${ }^{2}$ College of Computer and Communication Engineering, China University of Petroleum, Qingdao, Shandong 266580, China \\ ${ }^{3}$ Faculty of Engineering, Computing and Science, Swinburne University of Technology Sarawak Campus, 93350 Kuching, Malaysia
}

Correspondence should be addressed to Tao Song; taosong@hust.edu.cn

Received 19 May 2015; Accepted 16 September 2015

Academic Editor: Filippo de Monte

Copyright (C) 2015 Zhihua Chen et al. This is an open access article distributed under the Creative Commons Attribution License, which permits unrestricted use, distribution, and reproduction in any medium, provided the original work is properly cited.

Laser technology is vital in production of precision electronic components and has been widely used in modern industry. In laser soldering systems, accurate temperature control remains a challenging problem, since the temperature is highly sensitive to laser power and thermodynamic parameters of solder joints. In this paper, a good solution is proposed to solve the problem by controlling the temperature based on a novel thermodynamic model. In the model, many of the major factors are taken into account, such as laser energy, flux influence, and solder joints with different parameters. Aimed at the thermodynamic process and the influence of solder fluxes, a mixed mode control method is used to track the designed target temperature curve; this method can produce a solder joint with good quality. As a result, a model-based feed-forward and proportional, integral, and derivative (PID) mixed control method is developed. In practice, the proposed method is verified to have a wider product capability and production size; and a rate of good products of $99.94 \%$ is achieved with consuming approximately half of the energy comparing with manual soldering and constant laser power soldering.

\section{Introduction}

Soldering, which has a history of several thousand years dating back to $2500 \mathrm{BC}$, was considered as an art until the electronic age, when it was recognized as a technology [13]. With the development of the microelectronics industries, particularly optoelectronics and microelectromechanical systems (MEMS), the ever-increasing packaging density of components on printed board and the subsequent reduction in size continue to pose great challenges to manufacturers. Laser soldering has the advantages of low cost, high-density power, focused on a microregion, and low or no mechanical contact, which provide assembly with no damage to sensitive membranes in MEMS or optical components. Over the past few decades, the solder bumps are getting smaller and smaller such that the technical requirements for laser soldering have become stricter.

Simple thermal models of laser soldering (see, e.g., $[4,5]$ ) are used to describe the influence of material properties on the temperature within the solder joints, with different parameter settings producing different temperature profiles. Also, the requirements for a typical laser soldering system and the interaction of laser energy with the different materials used have been considered. The range of applicability of laser soldering is illustrated in $[3,6,7]$, where the understanding of heat transfer processes is essential to the successful application of the technique. Three-dimensional finite element models of laser soldering have been proposed to produce accurate predictions of temperature [8-11]. The factors involved in the laser soldering operations have been classed into three types, geometric, parametric, and temporal, which have been systemically discussed in $[8,9]$. Finite element models can show good agreement with experiment results such as the time-dependent pitch shift induced by the laser solder ball bonding process and single and multipass scanned beam soldering operations $[10,11]$. The mathematical model is verified by comparing simulation and experimental results. The model can correctly predict the temperature 


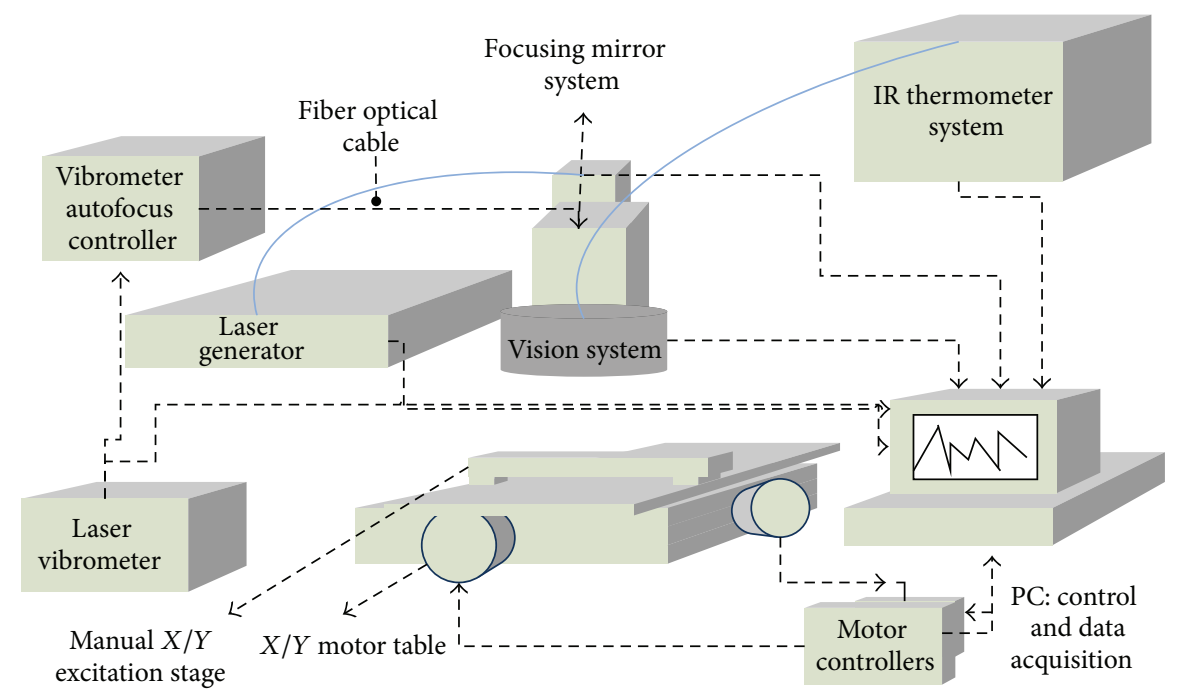

FIGURE 1: The laser soldering workstation with an IR thermometer system.

during soldering process but does not specify how to control the laser power to obtain a good solder joint. Meanwhile, a heat-flux based "building block" approach is also available; this method gives a sequential numerical form of the twodimensional transient Green's function solution equation based on a heat-flux formulation. It is appropriate for solving forward and inverse linear heat conduction problems and gives us guidance to our work [12].

Following the research line, plenty of possible control strategies or methods to control the laser power to track some target temperature curves have been developed. In general, noncontact detection sensors are used to measure the temperature of solder joints during the soldering process, which avoids polluting the joints. However, the measurement may be disturbed by hot volatile gas produced by the solder fluxes in solder paste and cause failed temperature measurement. In [13], effects of the solder fluxes, with respect to reflow duration and cleaning solvent residues on the catastrophic mirror damage, are investigated, but no explanation is imposed on how the solder fluxes affected temperature measurement. A significant controller should, in principle, control the laser power to match the solder joint temperature with the target temperature curve.

In this work, we propose a novel thermodynamic model to predict temperature variation during the laser soldering process, by which the parameters involved in the controller can be calculated through the model. Specifically, the temperature controller consists of an open-loop phase and a closedloop phase. In the open-loop phase, the laser power is given by the prediction of the proposed thermodynamic model; that is, a model-based feed-forward control method is used, since the temperature cannot be measured correctly because of the evaporation of solder fluxes when heated, whereas, in the closed-loop phase, when the solder fluxes have completely evaporated, the laser power is controlled by a mixed mode controller, including the model-based feed-forward and PID control. The simulation and experimental results show that the control method can successfully track the designed target temperature curve. As a result, stable and uniform solder joints can be acquired. In practice, the product yield rate achieves $99.94 \%$ with consuming approximately half of the energy comparing with manual soldering and constant laser power soldering. The proposed control method has also been verified to have a wider product capability and production size.

\section{Laser Soldering System}

A schematic of the laser soldering workstation is shown in Figure 1. A semiconductor laser beam is focused on the solder joint through a fiber and a focusing mirror. The reflected infrared from the solder joint is measured with an IR thermometer (type: OPTCT3MHSF, made in Germany, temperature range $100-600^{\circ} \mathrm{C}$ ). The $X-Y$ motion stage positions the sample board under the IR thermometer for each inspection location on the chip surface. A manual stage affixed to the top of the $X-Y$ stage is used for fine adjustment of the location of the excitation laser spot. A computer vision system captures fiducial marks on the sample substrate to provide precise alignment with the IR thermometer and excitation laser. The data acquisition, stage positioning, and vision systems are controlled by a computer, providing a fully automated inspection process. This system is capable of high measurement throughput with good accuracy at a relatively low cost. The commercial machine is shown in Figure 2; the following experiments are achieved in this workstation. For the first experiment, autofocusing is fixed and the laser beam diameter is manually adjusted to a size determined by the geometrical characteristics of the solder bonding pad. This requirement ensures that the solder joint is sufficiently heated at a fixed rate of energy loss. Hence, temperature variation caused by energy loss can be ignored.

The key parameters of the laser generator, including central wavelength of laser, and laser spot size (focus) are 


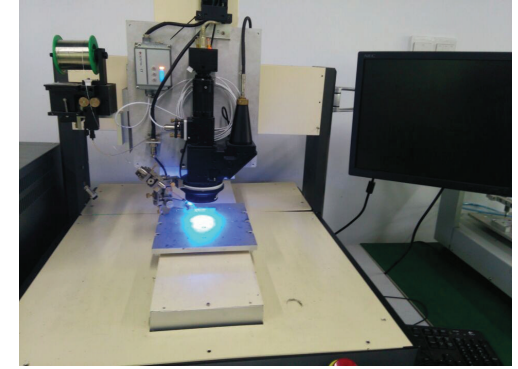

FIGURE 2: The machine of laser soldering and IR thermometer system.

given in Table 1. These parameters control the performance of the laser generator and determine whether the electronic elements are accurately welded.

The temperature control is used to make the temperature at the top centre of the soldering joint during the laser soldering process meet the target temperature curve through adjusting the laser power, by which a solder joint with good quality can be produced. The initial temperature of the solder joint is ambient temperature and a temperature rise from ambient temperature to $100^{\circ} \mathrm{C}$ is undetectable due to the range limit of the IR thermometer. Measurement from $100^{\circ} \mathrm{C}$ to the melt temperature (approximately $170^{\circ} \mathrm{C}$ ) is inaccurate due to the effects of evaporation of the solder fluxes when heated. After the evaporation, accurate measurement allows us to control the highest temperature and the rate of temperature change to obtain a good joint quality.

\section{Thermodynamic Modelling of Laser Soldering Process}

In this section, a thermodynamic model is built and verified.

In the thermodynamic model, an input and an output are assumed to describe the dynamic process of the system. The input is the controllable energy produced by the laser, while the output includes the heat absorbed by the solder paste that can cause the soldering joint temperature variation and the energy loss through heat conduction, convection, and radiation. Firstly, the mathematical formulation of the energy equation for calculating the temperature variation is introduced, and then two parameters are introduced into the equation, which are sensitive to the temperature variation. One is the geometrical factor associated with the shape of solder joint [9]; the other is the efficiency rate of the laser generator, which can decide the usage percentage of the energy. Subsequently, the thermodynamic equation is applied to the soldering process, which is divided into 4 stages according to the variation of the solder paste state. Based on the thermodynamic model, the temperature at the top centre of the soldering joint during the laser soldering process can be accurately predicted. Because the evaporation of solder fluxes when heated makes the temperature measurement incorrect, process identification is used to formulate the disturbed temperature measurement.
TABLE 1: The values of parameters of the laser generator.

\begin{tabular}{lc}
\hline Classes & SI value \\
\hline Fiber output power & $35.23 \mathrm{~W}$ \\
Central wavelength & $980 \pm 10 \mathrm{~nm}$ \\
Laser spot size (focus) & $0.4 \mathrm{~mm}$ \\
\hline
\end{tabular}

The mathematical model is verified through the comparison of simulation and experimental results. The matched results demonstrate that the model can correctly predict the temperature during the soldering process.

3.1. Energy Conservation Model of the Laser Soldering System. Heat transfer occurs from a region of high temperature to a region of low temperature, as described by the second law of thermodynamics. From the Fourier law of heat transfer, during an infinitely small time $d t$, heat energy $d Q_{c d}$ is produced when the medium passes through an infinitely small area, $d S$, along the normal, and is proportional to the medium temperature directional derivative, $\partial T / \partial n$, which points along the normal to the surface [14]:

$$
d Q_{c d}=-k(x, y, z) \frac{\partial T}{\partial n} d S d t
$$

where $k(x, y, z)$ is the coefficient of heat transfer of the medium at point $(x, y, z)$. The negative sign in (1) means that the heat energy transfers from the higher temperature side to the low.

For any closed surface, $f$, in the medium, $\mathscr{D}$, the area bounded by $f$ is denoted by $\Omega$. The heat transfer function from time $t_{1}$ to $t_{2}$ is

$$
d Q_{c d}=\int_{t_{1}}^{t_{2}}\left\{\iint_{f} k(x, y, z) \frac{\partial T}{\partial n} d S\right\} d t
$$

which can be deduced to

$$
d Q_{c d}=\int_{t_{1}}^{t_{2}} k(x, y, z)\left\{\iint_{f} \frac{\partial T}{\partial n} d S\right\} d t .
$$

It is assumed that the pad is uniform and, with the width $x$ and the length $y$, the partial differential equation can be approximated as a factor associated with its geometrical shape as shown by

$$
d Q_{c d}=\int_{t_{1}}^{t_{2}} K_{n n} \cdot K_{\text {shape }} \cdot T \cdot S d t,
$$

where $K_{n n}$ is the coefficient of heat conduction, $K_{\text {shape }}$ is the geometrical factor, and $S$ is the area of the bonding pad. Assume that $K_{\text {shape }}$ is associated with the value of $x$ and $y$ :

$$
K_{\text {shape }}=a\left(x^{2}+y^{2}\right)+b(x+y)+c,
$$

where parameters $a, b$, and $c$ describe the correlation between $K_{\text {shape }}$ and the width $x$ and length $y$, so it can be identified by experiments. 
TABLE 2: Calculated and experimental values of $K_{\text {shape }}$.

\begin{tabular}{lcc}
\hline Pad size $($ length $\times$ width $)$ & Calculated value & Experimental value \\
\hline $2.0 \times 1.0 \mathrm{~mm}$ & 23 & 23 \\
$1.5 \times 1.0 \mathrm{~mm}$ & 26.6 & 27 \\
$1.2 \times 0.6 \mathrm{~mm}$ & 32.4 & 33 \\
$0.8 \times 0.3 \mathrm{~mm}$ & 38.5 & 39
\end{tabular}

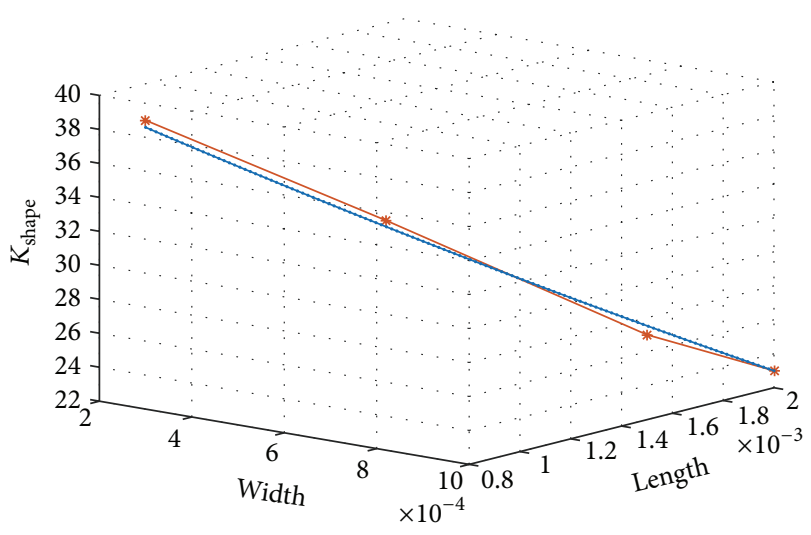

FIGURE 3: Relationship between length, width, and $K_{\text {shape }}$.

To identify the parameters, we designed a series of experiments, where

(i) laser power remains constant during the heating process;

(ii) pad size is altered in each experiment.

Based on the data sampled from the designed experiments, such as Data 1, Data 2, and Data 3 (shown in Figure 9), the parameter $K_{\text {shape }}$ in different pad size can be calculated from (15) (when the pad is heated with a fixed power, the pad will reach a steady temperature and $K_{\text {shape }}$ can be worked out as $\left.T(\infty)=q_{1} / p_{1}\right)$. The pads with different sizes and the corresponding $K_{\text {shape }}$ values are shown in Table 2 . According to the distribution of $K_{\text {shape }}$ along the $x$-axis and $y$-axis, we can identify the parameters $a, b$, and $c$ used in (5) by the method of least squares. The line shown in Figure 3 is fitted by the method of least squares. Then, we can calculate $K_{\text {shape }}$ from (5) and the values calculated from (5) are also shown in Table 2.

When solder paste is heated, a regular shape will be formed, and this ensures that the geometric characteristics of the solder paste change little during the soldering process. Thus, $K_{\text {shape }}$ can be assumed to be constant.

Heat convection occurs when bulk flow of fluid (gas or liquid) carries heat along with the fluid. Convective processes also transfer heat partly by diffusion. Forced convection is where the fluid flow is forced by use of a pump, fan, or other mechanical means; that is,

$$
Q_{\mathrm{cv}}=\int_{t_{1}}^{t_{2}} h_{f}\left(T-T_{B}\right) S d t,
$$

where $h_{f}$ is the coefficient of convective heat transfer and $T_{B}$ is the indoor temperature. Thermal radiation occurs through vacuum or any transparent medium (solid or fluid). The energy transfers by means of photons in electromagnetic waves:

$$
Q_{\mathrm{ra}}=\varepsilon \sigma A_{1} F_{12}\left(T_{1}^{4}-T_{2}^{4}\right) \text {, }
$$

where $Q_{\mathrm{ra}}$ is the rate of heat flow; $\varepsilon$ is the blackbody radiation; $\sigma=5.67 \times 10^{-8} \mathrm{~W} / \mathrm{m}^{2} \mathrm{~K}^{4}$ is the Stephen-Boltzmann constant; $A_{1}$ is the area of radiant surface $1 ; F_{12}$ is the shape factor from radiant surface 1 to surface $2 ; T_{1}$ and $T_{2}$ are the absolute temperature of radiant surfaces 1 and 2 , respectively $\left(T_{1}=\right.$ $273+200, T_{2}=273$ which are temperatures from Kelvin rating to degree Celsius). Consider

$$
\begin{aligned}
Q_{\mathrm{ra}}= & \varepsilon \sigma A_{1} F_{12}\left(T_{1}^{4}-T_{2}^{4}\right) \\
\approx & 5.68 \times 10^{-8} \times \sigma \times 10^{-6} \times\left(473^{4}-273^{4}\right) \times 4 \\
& \times F_{12} \approx 2 \times 10^{-3} \times F_{12} \ll 1 .
\end{aligned}
$$

Since $Q_{\text {ra }} \ll 1$, it can be ignored.

Energy, including thermal energy, is moved by the physical transfer of a hot or cold object from one place to another. A practical example is thermal hydraulics. The specific heat is the amount of heat per unit mass required to raise the temperature by one degree Celsius. The heat and temperature change can be usually expressed as

$$
Q_{\text {heat }}=C \cdot m \cdot \Delta T \text {, }
$$

where $C$ is the specific heat capacity, $m$ is the mass of the material, and $\Delta T$ is the temperature variation during the heat process. The phase change can be ignored, since the heat added or removed during a phase change process will not change the temperature.

We apply these relationships to laser soldering system. The energy flow at the same phase can be expressed as follows:

$$
\begin{aligned}
\int W K_{\text {laser }} d t= & \int K_{n n} K_{\text {shape }} T S d t+h_{f}\left(T-T_{i}\right) S \\
& +m C_{i}\left(T-T_{i}\right),
\end{aligned}
$$

where $i$ denotes the metallographic phase, $T_{i}$ is the initial temperature of phase $i$, and $K_{\text {laser }}$ is the efficiency rate of the laser generator.

3.2. The Thermodynamic Model of Laser Soldering Process. The laser soldering process is divided into 4 stages corresponding to the metallographic phases of solder past: preheating, active, reflowing, and cooling.

In laser soldering process, the entire filler material needs to be firstly heated to its melting temperature. The temperature should be strictly controlled during the heating process to ensure a high quality joint. When an IR thermometer is used to measure the joint temperature, the temperature may be controlled by closed-loop feedback controller, including laser power, interaction time, laser spot size, and laser beam displacement [15]. In our case, the energy is brought to the solder paste by direct radiation. The laser energy needed to 
TABLE 3: Parameters involved in the model.

\begin{tabular}{lcc}
\hline Symbol & Quantity & SI value \\
\hline$C_{1}$ & Specific heat capacity of the paste before melting & $230 \mathrm{~kJ} / \mathrm{kg} /{ }^{\circ} \mathrm{C}$ \\
$C_{2}$ & Specific heat capacity of the paste after melting & $200 \mathrm{~kJ} / \mathrm{kg} /{ }^{\circ} \mathrm{C}$ \\
$C_{3}$ & Specific heat capacity of the pad & $230 \mathrm{~kJ} / \mathrm{kg} /{ }^{\circ} \mathrm{C}$ \\
$m$ & Mass of the pad & $\mathrm{kg}$ \\
$m_{1}$ & Mass of the solder paste \\
$T_{B}$ & Indoor temperature & $25^{\circ} \mathrm{C}$ \\
$S$ & Area of the pad & $2.4 \times 10^{-6} \mathrm{~m}^{2}$ \\
$K_{\text {laser }}$ & Laser absorptivity & 0.77 \\
$K_{\text {shape }}$ & Geometrical factor & 38.5 \\
$K_{\text {nn }}$ & Coefficient of heat conduction of the paste \\
$h_{f}$ & Coefficient of convective heat transfer & $401 \mathrm{~W} / \mathrm{m}^{2} /{ }^{\circ} \mathrm{C}$ \\
$T_{\text {melt }}$ & Melting temperature of the solder paste & $10 \mathrm{~W} / \mathrm{m}^{2}$ \\
$T_{\text {top }}$ & Maximum temperature of the laser soldering process & $172^{\circ} \mathrm{C}$ \\
$T(j)$ & Temperature of the joint at time $j$ & ${ }^{\circ} \mathrm{C}$ \\
$Q_{\text {melt }}$ & Latent heat energy for melting solder & ${ }^{\circ} \mathrm{C}$ \\
\hline
\end{tabular}

melt the filler material depends on the materials, the geometry of the underlays, and the contact condition with the pad.

The parameters used in the mathematical model are explained in Table 3.

The thermodynamic equation in the 4 stages is as follows:

(1) When the temperature is under the paste melt temperature $\left(T<T_{\text {melt }}\right)$, the energy conservation equation is as follows:

$$
\begin{aligned}
\int W K_{\text {laser }} d t= & \int K_{n n} K_{\text {shape }} T S d t+h_{f}\left(T-T_{B}\right) S \\
& +m C_{1}\left(T-T_{B}\right) .
\end{aligned}
$$

The thermodynamic model for the heating process can be obtained from the energy conservation equation (11) by taking the time derivative:

$$
W K_{\text {laser }}=K_{n n} K_{\text {shape }} T S+h_{f} T S+m C_{1} \dot{T} \text {. }
$$

The initial temperature of the system, $T(0)$, is ambient indoor temperature, $T_{B}$, so (12) can be derived as

$$
\dot{T}+\frac{K_{n n} K_{\text {shape }} S}{m C_{1}+h_{f} S} T=\frac{W K_{\text {laser }}}{m C_{1}+h_{f} S} .
$$

To simplify matters, define $p_{1}=K_{n n} K_{\text {shape }} S /\left(m C_{1}+\right.$ $\left.h_{f} S\right)$ and $q_{1}=W K_{\text {laser }} /\left(m C_{1}+h_{f} S\right)$; then (13) can be reduced to

$$
\dot{T}+p_{1} T=q_{1}
$$

The solution can be derived from (14):

$$
T(t)=\left(T_{B}-\frac{q_{1}}{p_{1}}\right) e^{-p_{1} t}+\frac{q_{1}}{p_{1}} .
$$

(2) During the solder paste melting phase $\left(T=T_{\text {melt }}\right)$, the temperature remains constant. The absorbed energy $Q_{\text {melt }}$ is used to melt the solder paste, and the time $t_{1}$ to melt the paste can be expressed as

$$
t_{1}=\frac{Q_{\text {melt }} m_{1}}{\left(W K_{\text {laser }}\right)},
$$

where $Q_{\text {melt }}, W$, and $K_{\text {laser }}$ are given in Table 3.

(3) After the paste is melted $\left(T>T_{\text {melt }}\right)$, an alloy is formed from the mixture of substances, and the heat capacity changes. The temperature and the energy follow (11), where the initial temperature, $T(0)$, is the melt temperature, $T_{\text {melt }}$ :

$$
\begin{aligned}
\int W K_{\text {laser }} d t= & \int K_{n n} K_{\text {shape }} T S d t+h_{f}\left(T-T_{\text {melt }}\right) S \\
& +m C_{2}\left(T-T_{\text {melt }}\right) .
\end{aligned}
$$

The maximum temperature is $T_{\text {top }}$ which appears at the end of this stage.

(4) After the heating process $\left(T_{\text {melt }}<T\right)$, the laser is shut down and the energy conservation equation is

$$
\begin{gathered}
\int K_{n n} K_{\text {shape }} T S d t+h_{f}\left(T-T_{\text {melt }}\right) S \\
+m C_{2}\left(T-T_{\text {melt }}\right)=0 .
\end{gathered}
$$

The thermodynamic model also can be obtained from the energy conservation equation (19) by taking the time derivative:

$$
\dot{T}+\frac{K_{n n} K_{\text {shape }} S}{m C_{2}+h_{f} S} T=0 .
$$




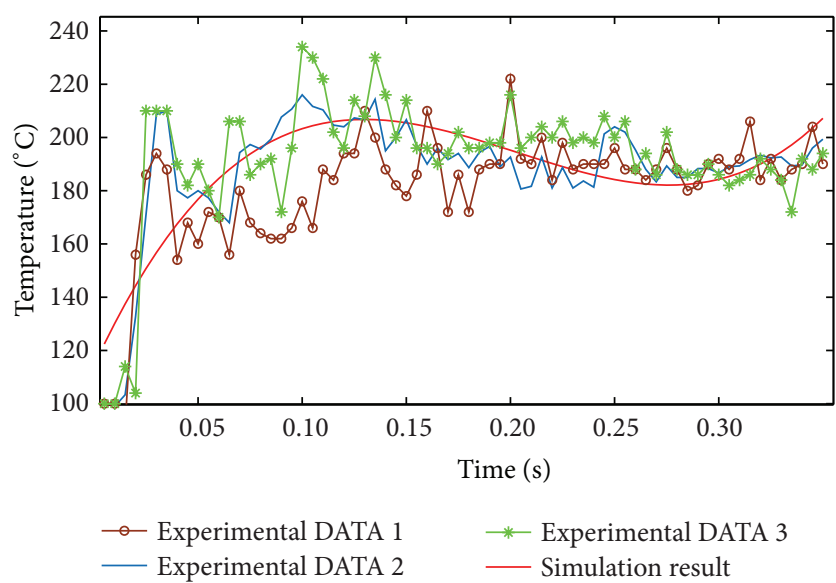

Figure 4: Simulated and experimental temperature measures in the presence of solder fluxes.

We have $T(0)=T_{\text {top }}$, and define $p_{2}=K_{n n} K_{\text {shape }} S /$ $\left(m C_{2}+h_{f} S\right)$; the temperature can be derived from (19) as

$$
T(t)=T_{\text {top }} e^{-p_{2} t}
$$

3.3. Effect of the Solder Fluxes. When heated, the solder fluxes evaporate and form hot gas at a high rate, which greatly influences the temperature measurement by the IR thermometer (as mentioned in Section 2). If a feedback control method is applied to the system, the failed temperature measurement may lead to system instability.

In this paper, the evaporation process of the solder fluxes is treated as a disturbance process to the normal laser soldering process. The measured temperature data during the evaporation process is shown in Figure 4, and 3 experiments under the fixed laser power are carried out to measure the temperature variation during the evaporation of fluxes. The process data is used to identify the evaporation model. The evaporation process can be identified through a third-order polynomial:

$$
T_{\text {paste }}(t)=\left(d_{0} t^{3}+d_{1} t^{2}+d_{2} t+d_{3}\right) P,
$$

where $T_{\text {paste }}(t)$ is the expected temperature of the paste at time $t ; d_{0}, d_{1}, d_{2}$, and $d_{3}$ are parameters to be identified; and $P$ is the power of the laser.

The parameters are identified in Figure 4, which also shows the agreement between simulation and experimental measurement. The results illustrate that (21) can formulate the dynamic evaporating process.

\section{Control Strategy for Laser Soldering Process}

According to the structure of the laser soldering system and the characteristics of the solder joint, it is necessary to design a target temperature curve which can result in a good quality of solder joint and to develop a fine temperature control method to track the target temperature curve. As discussed above, the temperature feedback control cannot be

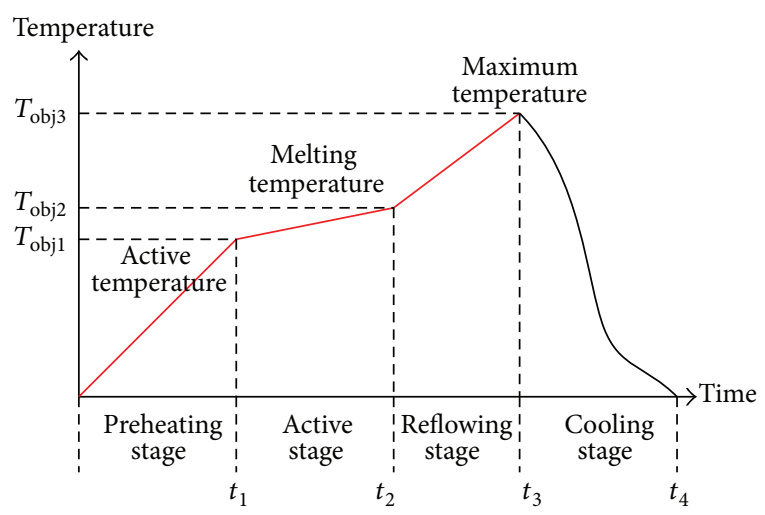

FIGURE 5: The piecewise linear curve of the target temperature curve.

directly applied to track the target temperature because of the evaporation of solder fluxes. An open-loop and closedloop mixed control strategy is proposed to track the target temperature. A model-based open-loop control method is used to achieve the target temperature in the preheating stage avoiding the errors of temperature measurement caused by the evaporation of solder fluxes. After the evaporation of solder fluxes, that is, in the active and reflowing stages, a model-based feed-forward and PID mixed controller is used to track the target temperature curve, since the temperature measurement is reliable without the evaporation of solder fluxes.

4.1. Target Temperature Curve Designing. Based on practical experiences, the temperature, rate of temperature change, and heating duration time are key factors to obtain a solder joint with good quality. The soldering process can be divided into 4 stages: preheating, active, reflowing, and cooling stages. So a target temperature curve is designed into 4 parts according to the requirement in different stages. A target temperature curve is shown in Figure 5.

The piecewise linear curve is described as follows:

$$
\begin{aligned}
& T_{\text {obj }}(t) \\
& \quad= \begin{cases}T_{\mathrm{obj} 1} \cdot \frac{t 1}{t} & 0 \leq t \leq t 1, \\
T_{\mathrm{obj} 1}+\frac{\left(T_{\mathrm{obj} 2}-T_{\mathrm{obj} 1}\right)(t-t 1)}{(t 2-t 1)} & t 1<t \leq t 2, \\
T_{\mathrm{obj} 2}+\frac{\left(T_{\mathrm{obj} 3}-T_{\mathrm{obj} 2}\right)(t-t 2)}{(t 3-t 2)} & t 2<t \leq t 3,\end{cases}
\end{aligned}
$$

where $T_{\text {obj1 }}, T_{\text {obj2 } 2}$, and $T_{\text {obj3 }}$ are expected temperatures in different stages; $t 1, t 2, t 3$, and $t 4$ are time nodes distinguishing different stages; $t$ is the time variable.

During the preheating stage $(0 \leq t \leq t 1)$, laser energy is used to heat the welding pad and the solder paste until the active temperature is reached (the temperature that the solvent agent activates to remove oxidation and increase the spreading area of solder paste). In the heating process, the rate of temperature change must be in a limited range (the range can be obtained by experiments). Otherwise, a too high rate 
can damage the welding pad and deprive the solvent from solder paste; if the rate of temperature change is too small, it cannot stimulate the activity of the solvent. The loss of solvent can cause the solder paste splashing, which can result in short circuit.

The active stage ( $t 1<t \leq t 2)$ is to make the rate of temperature change of the heated welding pad be consistent with the heated solder paste; otherwise, poor adherence can occur because of the different absorbance capabilities between the welding pad and solder paste. In the active stage, the solvent in the solder paste is fully activated and the temperature is raised to $T_{\mathrm{obj} 2}$. The duration time of this stage is the key factor for a joint with good quality. If the duration time of active stage is too long, the welding pad may be deformed and the pin and pad may be oxidized. Instead, if the duration is too short, the heat may be distributed unevenly; then the shear resistance of the final joint may be poor, and porosity may form in the final solder joint.

When the reflowing stage $(t 2<t \leq t 3)$ starts, solder paste has transformed to liquid alloy and an early shape of solder joint has formed by the spread of liquid alloy. In the reflowing stage, suitable $T_{\text {obj } 3}$ is required to weaken the viscosity and interfacial tension of the paste. $T_{\mathrm{obj} 3}$ and the rate of temperature change are key factors. Too high temperature may damage the pad and components and accelerate the oxidation of solder paste and welding pad. In contrast, too low temperature may weaken the efficiency of the solvent and increase the possibility of inner voids.

When the heating process is completed, the cooling stage ( $t 3<t \leq t 4)$ begins. The laser is switched off, and the solder joint is finally formed. The laser soldering process is completed.

But, in fact, the target temperature curve (Figure 5) cannot be directly used as a reference in practical laser soldering systems, because the rate of change of laser power caused by the rate of change of target temperature always leads to the practical temperature instability, which often results in solder joints thermal breakdown or solder paste splashing. In this paper, the target temperature curve (Figure 5) is used as the input in simulation instead of in practical system, while the output of the simulation (see Figure 6) is used as the reference of practical laser solder system. The temperature produced by simulation is more continuous and smooth comparing to Figure 5, which can result in a better quality.

By this way, the responding laser power produced by the same simulation can serve as the feed-forward control signal, which will not be affected by the measurement noise. Based on the designing of the temperature reference and the feed-forward control signal, the control structure and control method is developed in the next section.

4.2. Control Method. The complete block diagram of control system is shown in Figure 7, where the dead-time controller is used to switch the open-loop and closed-loop control method during different solder stages. In the preheating stage, the laser power is only given by the feed-forward control signal regardless of the temperature measurement. In the active and



FIgURE 6: The temperature produced by simulation.

reflowing stages, the laser power is also given by the feedforward control and the PID controller.

The description of control process is detailed as follows.

When the parameters $T_{\text {obj1 }}, T_{\text {obj2 }}, T_{\text {obj3 }}, t 1, t 2$, and $t 3$ are given by experiences or experiments, the target temperature $T_{\text {obj }}(t)$ at time $t$ can be determined. Some variables are defined as follows to make the control process clear:

(i) $T s(t)$ : the output temperature in simulation at time $t$.

(ii) $\operatorname{Tr}(t)$ : the real-time temperature of the solder joints at time $t$.

(iii) $\Delta T(t)$ : the temperature difference between the realtime output and the simulated output.

(iv) $\Delta W(t)$ : the power of laser generator at time $t$, which is given by PID controller 1 .

(v) $W s(t)$ : the power of laser generator at time $t$, which is given by PID controller 2 in simulation.

(vi) $W f(t)$ : the feed-forward control signal at time $t$, which is equal to $W s(t)$; that is, $W f(t)=W s(t)$.

(vii) $W r(t)$ : the practical laser generator at time $t$, which is $W r(t)=\Delta W(t)+W f(t)=\Delta W(t)+W s(t)$.

During the preheating stage, the dead-time controller makes the PID controller 1 ineffective, which means $\Delta W(t)=$ 0 , and $W r(t)=W f(t)=W s(t)$. In this stage, the practical temperature is controlled by an open-loop method, and the control signal is given by simulation. So the evaporation of the solder fluxes cannot influence the temperature control process.

During the active and reflowing stages, the dead-time controller makes the PID controller 1 effective and the practical laser power is $W r(t)=\Delta W(t)+W f(t)=\Delta W(t)+$ $W s(t)$. Then, a closed-loop and feed-forward mixed control method is used to track the target temperature, which can trace the given temperature more finely.

When the cooling stage starts, the laser power shuts down and the solder joint goes into a passive cooling process. The solder joint forms ultimately in the end of this stage. 


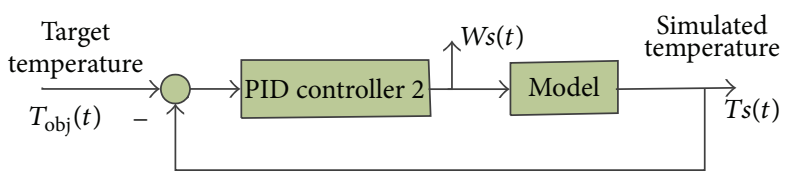

(a) Simulation system

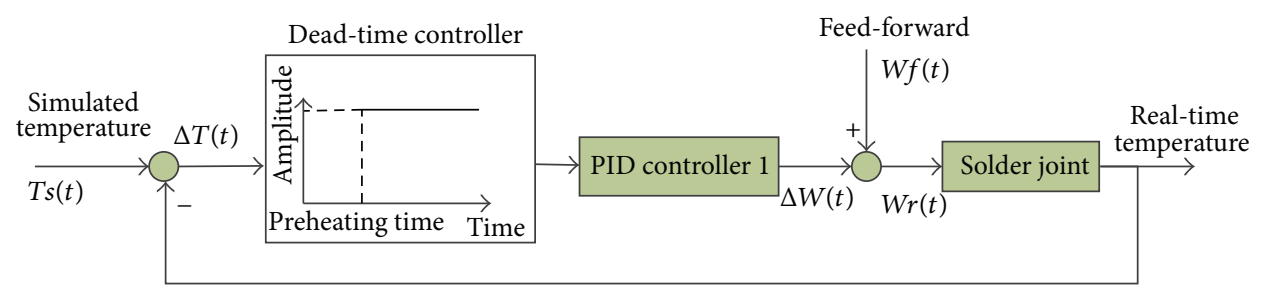

(b) Practical laser soldering system

FIGURE 7: Control structure for practical laser soldering system.



FIgure 8: Structure of the CMOS Camera Module.

\section{Experimental and Simulation Results of Model Verification and Control Strategy Application}

In these experiments, $\mathrm{Sn}_{64} \mathrm{Bi}_{35} \mathrm{Ag}_{1}$ (wt.\%) lead-free soldering paste was used in order to assemble two components. Figure 8 shows the assembly structure, including one $8.5 \times 8.5 \times 6.5 \mathrm{~mm}$ sensor and one $0.02 \mathrm{~mm}$ thick stainless substrate. There are two $0.8 \times 0.3 \times 0.018 \mathrm{~mm} \mathrm{Cu}$ pads with $\mathrm{Au} / \mathrm{Ni}$ metallization layer on the $\mathrm{Cu}$ pads surface pasted on the surface of the substrate. In front of the sensor, there are two stainless steel feets with $0.1 \mu \mathrm{m}$ thick $\mathrm{Au} / \mathrm{Ni}$ metallization layer, which are the two components of CMOS Camera Module.

The laser soldering system used in this experiment is shown in Figures 1 and 2, which is a semiconductor laser $(\lambda=980 \mathrm{~nm})$, equipped with a $200 \mathrm{~nm}$ diameter optical fibre, and the usable power of the laser is not higher than $32 \mathrm{~W}$. The laser beam is motionless and its spot on the top composite part can be adjusted from $50 \mathrm{~nm}$ to $400 \mathrm{~nm}$ diameter. The top surface of the soldering joint is placed below the focal plane of the laser beam. An IR thermometer is used to measure the temperature at the top centre of the soldering joint during the laser soldering process, which will be compared to the thermal simulations.

5.1. Validation of the Soldering Process in a Constant Power. To validate the correction of $K_{\text {shape }}$ based thermodynamic

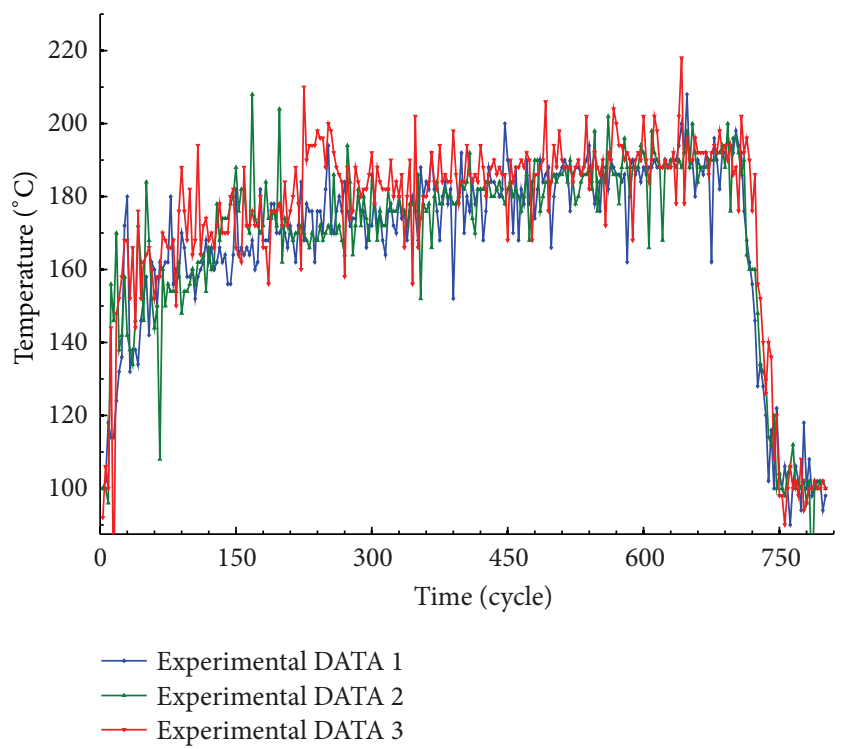

FIGURE 9: Temperature variation curves during the laser soldering process.

model (as seen in (5)), the soldering process in a constant power is validated firstly. In this experiment, the laser power is fixed to $15 \mathrm{~W}$, and the duration is 700 duty cycles.

Figure 9 shows the temporal evolution of temperature, which is measured at the top centre of the solder paste using the IR thermometer with temperature acquisition starting at $100^{\circ} \mathrm{C}$.

When the effect of the solder fluxes evaporation is considered, the final model of evaporation process is (21).

Because the evaporation of solder fluxes brings about the errors of measurement, the first part of the curves (before the 50th cycle) is mismatched (see Figure 10). However, the peak value of the evaporation temperature is accurately predicted. After the evaporation process of solder fluxes, the simulation matches the experimental data well during the second part of the curve (after the 50th cycle). 
TABle 4: Comparison of work efficiency.

\begin{tabular}{lcccc}
\hline Method & $\begin{array}{c}\text { Time } \\
(1000 \text { samples })\end{array}$ & $\begin{array}{c}\text { Energy } \\
\text { consumption } \\
(100 \text { samples })\end{array}$ & $\begin{array}{c}\text { Rate of } \\
\text { good product } \\
(1000 \text { samples })\end{array}$ & $\begin{array}{c}\text { Restriction on } \\
\text { production size }\end{array}$ \\
\hline Manual & $400 \mathrm{~s}$ & $14 \mathrm{~kJ}$ & $95.66 \%$ & $\geq 1 \times 0.8 \mathrm{~mm}$ \\
Traditional control & $700 \mathrm{~s}$ & $10.5 \mathrm{~kJ}$ & $91.37 \%$ & $\geq 0.6 \times 0.3 \mathrm{~mm}$ \\
The proposed method & $800 \mathrm{~s}$ & $6 \mathrm{~kJ}$ & $99.94 \%$ & $\geq 0.3 \times 0.3 \mathrm{~mm}$ \\
\hline
\end{tabular}



Figure 10: Simulation and experimental results.

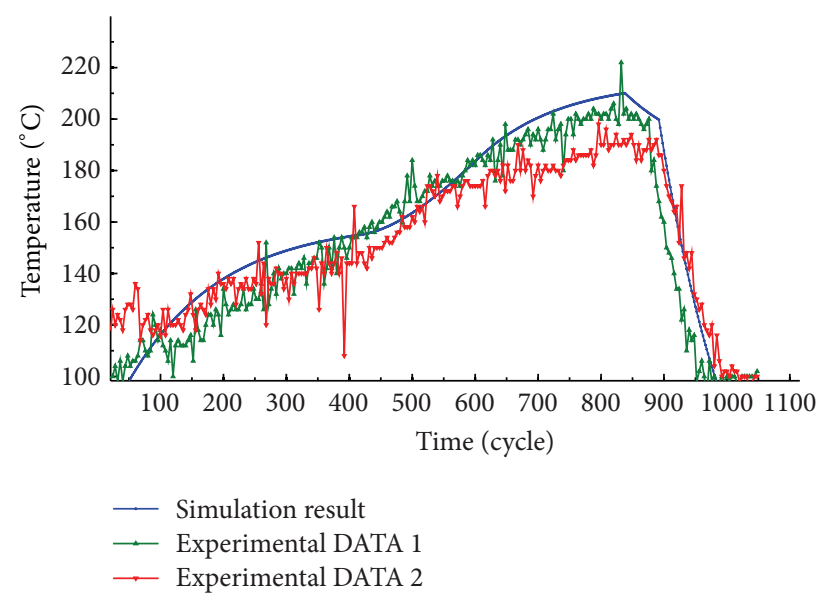

FIGURE 11: Experimental and simulated results.

The thermodynamic models (as (21)) give the relationship between temperature variation and laser power, which can be applied in soldering process to achieve a fine temperature control.

5.2. Experiments and Analysis. Experiments and simulations are compared in Figure 11. There is a good agreement between the simulation and experiments. The soldered joint of CCM using the proposed control method is shown in Figure 12. The solder paste climbs to the top of the pin and the surface of the joint is smooth and bright without solder residue or splash,

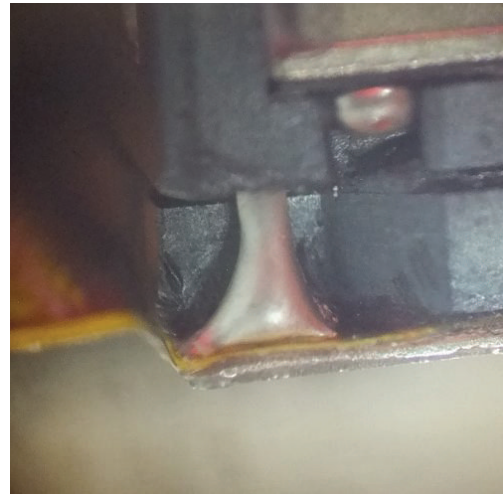

FIGURE 12: Soldering result.

and the joint illustrates the good effect by the proposed fine temperature control method.

For an illustrative purpose, three soldering methods are compared in 4 aspects, time, energy consumption, rate of good products, and restriction on production size, that is, the manual welding ( $35 \mathrm{~W}$ electric soldering irons used by skilled laborers), traditional laser soldering (with constant temperature), and the proposed method. In the experiments, we use each method to weld 1000 solder joint samples and the results are shown in Table 4.

Though manual welding has a high rate of good product and takes the least time, it consumes more power and the production size is very limited and the gas produced in the welding process may impair the health of human. The proposed control method has evident advantages in decreasing power consumption, improving quality, and widening the range of production sizes.

\section{Conclusion}

A thermodynamic model is set up to analyze the relation between the joint temperature and laser power during the laser soldering process. Based on the model, the solder temperature in the soldering process is accurately predicted. According to the prediction, an open-loop and closed-loop mixed control strategy is proposed to track the target temperature curve, since the closed-loop control cannot directly apply to the laser solder system because of the evaporation of the solder fluxes during the preheating stage. The simulation and experiment results match well with each other; thus, the thermodynamic model is verified. A dead-time controller is used to switch the open-loop and closed-loop control 
method. To achieve a better quality of solder joint, the simulation output temperature is used as the practical target temperature, which is continuous and smooth. In this way, the laser power produced by simulation can be used as the feed-forward control signal minimizing the effect of measure noise. By comparison, the proposed control method has evident advantages in decreasing power consumption, improving quality, and widening the range of production sizes. The proposed control method gives a feasible way to solder very small products. In future work, more accurate estimation for the geometry coefficient $K_{\text {shape }}$ is needed to be done and the target temperature is needed to be achieved by scientific quantizing.

For further research, it is worth using neural-like computing models (see, e.g., [16-18]) and intelligent algorithms, such as GA and EM [19], to develop intelligent temperature control method, particularly online control method, for laser soldering systems. Low-dispersion sequences were proposed in detecting meaningful patterns in [20]; it is worth using it in method of controlling temperature in laser soldering systems.

Another important researching direction is to use a heatflux based "building block" approach to control temperature in our system. In the method, it can use a sequential numerical form of the two-dimensional transient Green's function solution equation based on a heat-flux formulation, thus achieving the function of temperature prediction and regulation (see, e.g., [12]).

\section{Conflict of Interests}

The authors declare that there is no conflict of interests regarding the publication of this paper.

\section{Acknowledgment}

The authors thank the financial support for this work from the Chinese National Natural Science Foundation (61170183, 61272093, 61272022, 61272071, 61370105, 61374139, 61402187, 61572522, and 61572523).

\section{References}

[1] S. M. Naveed and R. L. Woods, "Diode laser soldering: a lumped parameter mathematical model and comparison of different optical soldering technologies," in High-Power Diode Laser Technology and Applications, vol. 4973 of Proceedings of SPIE, International Society for Optics and Photonics, San Jose, Calif, USA, January 2003.

[2] W.-K. Chen, Linear Networks and Systems, Wadsworth Publishing, Belmont, Calif, USA, 1993.

[3] P. M. Beckett, A. R. Fleming, R. J. Foster, J. M. Gilbert, and D. G. Whitehead, "The application of semiconductor diode lasers to the soldering of electronic components," Optical and Quantum Electronics, vol. 27, no. 12, pp. 1303-1311, 1995.

[4] J. Nicolics, L. Musiejovsky, and E. Semerad, "Optimization of process parameters for laser soldering of surface mounted devices," IEEE Transactions, Components, Hybrids, and Manufacturing Technology, vol. 15, no. 6, pp. 1155-1159, 1992.
[5] J. Nicolics and G. Hobler, "Numerical analysis of transient temperature distributions during laser soldering," COMPELThe International Journal for Computation and Mathematics in Electrical and Electronic Engineering, vol. 13, no. 4, pp. 845-860, 1994.

[6] P. M. Beckett, A. R. Fleming, J. M. Gilbert, and D. G. Whitehead, "Practical application of laser soldering in electronics assembly," in Proceedings of the IEEE International Symposium on Industrial Electronics (ISIE '97), pp. 59-64, July 1997.

[7] P. M. Beckett, A. R. Fleming, J. M. Gilbert, and D. G. Whitehead, "The laser in manufacture-its use in the soldering of electronic assemblies," Transactions of the Institute of Measurement and Control, vol. 21, no. 1, pp. 2-7, 1999.

[8] M. R. Frewin and D. A. Scott, "Finite element model of pulsed laser welding," Welding Journal, vol. 78, no. 15, 1999.

[9] P. M. Beckett, A. R. Fleming, J. M. Gilbert, and D. G. Whitehead, "The finite element modelling of laser soldering for electronic assemblies," International Journal of Numerical Modelling: Electronic Networks, Devices and Fields, vol. 15, no. 3, pp. 265-281, 2002.

[10] P. M. Beckett, A. R. Fleming, J. M. Gilbert, and D. G. Whitehead, "Numerical modelling of scanned beam laser soldering of fine pitch packages," Soldering and Surface Mount Technology, vol. 14, no. 1, pp. 24-29, 2002.

[11] D. Tian, C. Wang, and Y. Tian, "Modeling of micropitch Shift of a magnetoelectrical sensor during laser solder ball bonding process," IEEE Transactions on Advanced Packaging, vol. 32, no. 1, pp. 136-145, 2009.

[12] F. De Monte, J. V. Beck, and D. E. Amos, "A heat-flux based 'building block' approach for solving heat conduction problems," International Journal of Heat and Mass Transfer, vol. 54, no. 13-14, pp. 2789-2800, 2011.

[13] P. Zhalefar, A. Dadoo, M. Nazerian et al., "Study on effects of solder fluxes on catastrophic mirror damages during laser diode packaging," IEEE Transactions on Components, Packaging and Manufacturing Technology, vol. 3, no. 1, pp. 46-51, 2013.

[14] Y. Tian, C. Wang, and D. Liu, "Thermomechanical behaviour of PBGA package during laser and hot air reflow soldering," Modelling and Simulation in Materials Science and Engineering, vol. 12, no. 2, pp. 235-243, 2004.

[15] C. Chaminade, E. Fogarassy, and D. Boisselier, "Diode laser soldering using a lead-free filler material for electronic packaging structures," Applied Surface Science, vol. 252, no. 13, pp. 44064410, 2006.

[16] T. Song, L. Pan, and G. Păun, "Asynchronous spiking neural P systems with local synchronization," Information Sciences, vol. 219, pp. 197-207, 2013.

[17] T. Song and L. Pan, "Spiking neural P systems with rules on synapses working in maximum spikes consumption strategy," IEEE Transactions on NanoBioscience, vol. 14, no. 1, pp. 38-44, 2015.

[18] T. Song and L. Pan, "Spiking neural P systems with rules on synapses working in maximum spiking strateg," IEEE Transactions on NanoBioscience, vol. 14, no. 4, pp. 465-477, 2015.

[19] X. Wang and Y. Miao, "GAEM: a hybrid algorithm incorporating GA with EM for planted edited motif finding problem," Current Bioinformatics, vol. 9, no. 5, pp. 463-469, 2014.

[20] X. Wang, Y. Miao, and M. Cheng, "Finding motifs in DNA sequences using low-dispersion sequences," Journal of Computational Biology, vol. 21, no. 4, pp. 320-329, 2014. 




Advances in

Operations Research

mansans

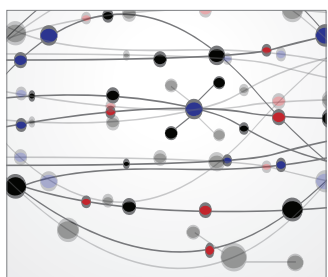

The Scientific World Journal
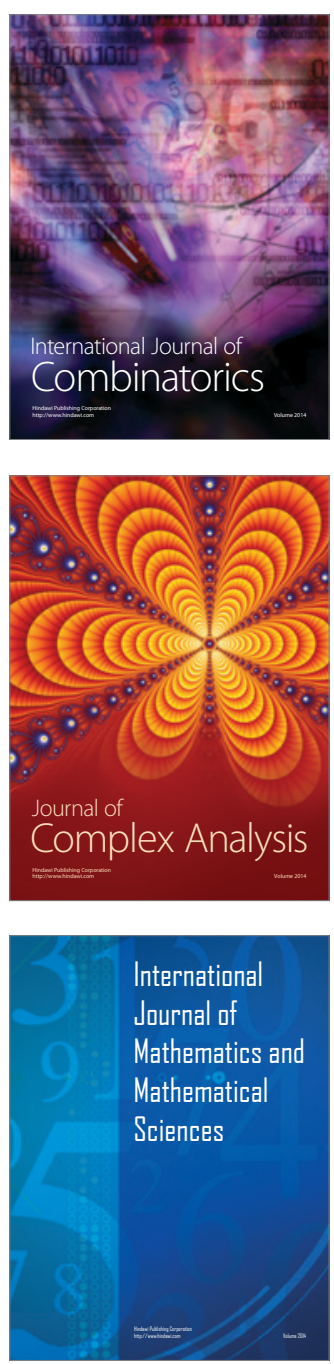
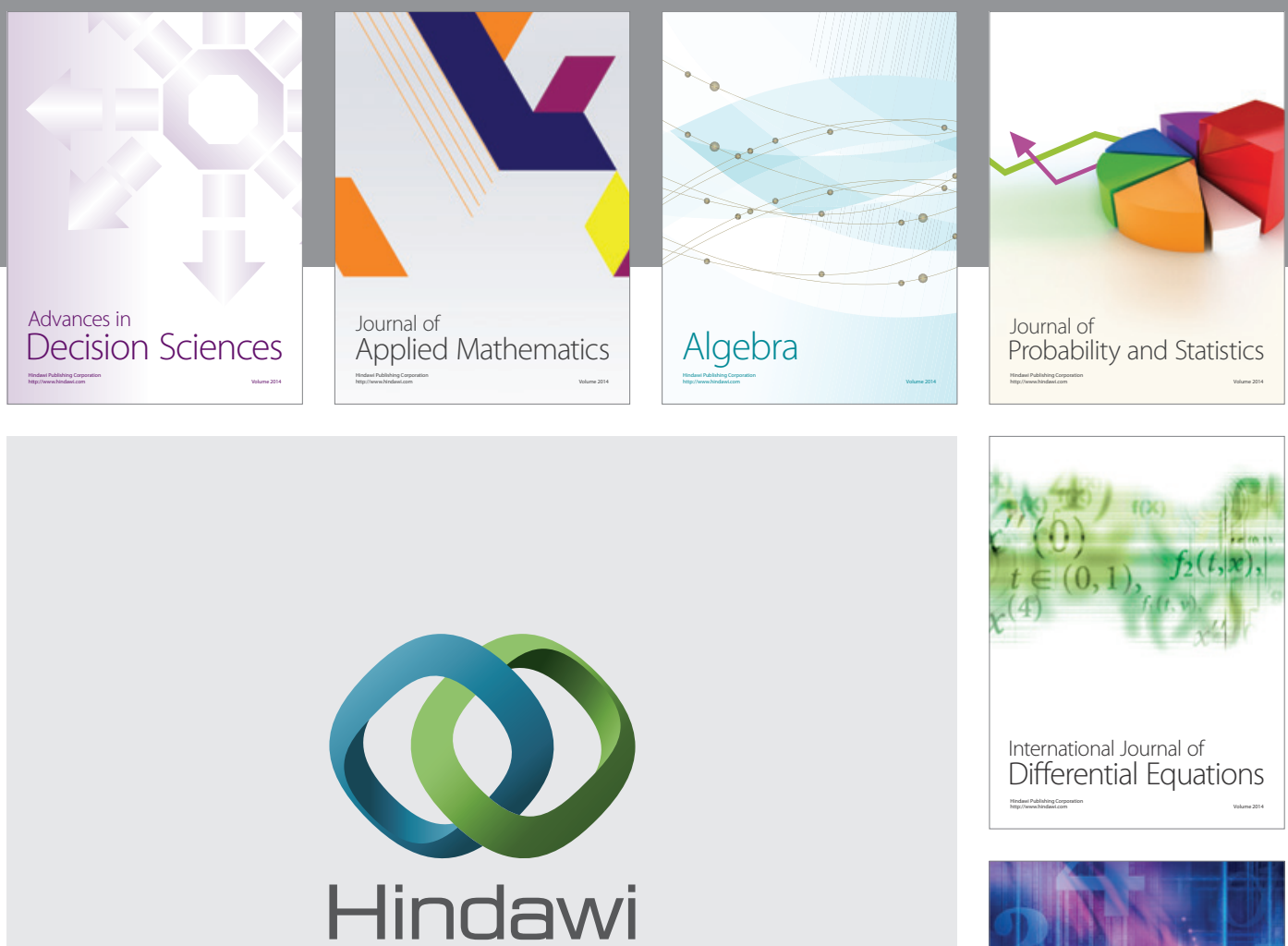

Submit your manuscripts at http://www.hindawi.com
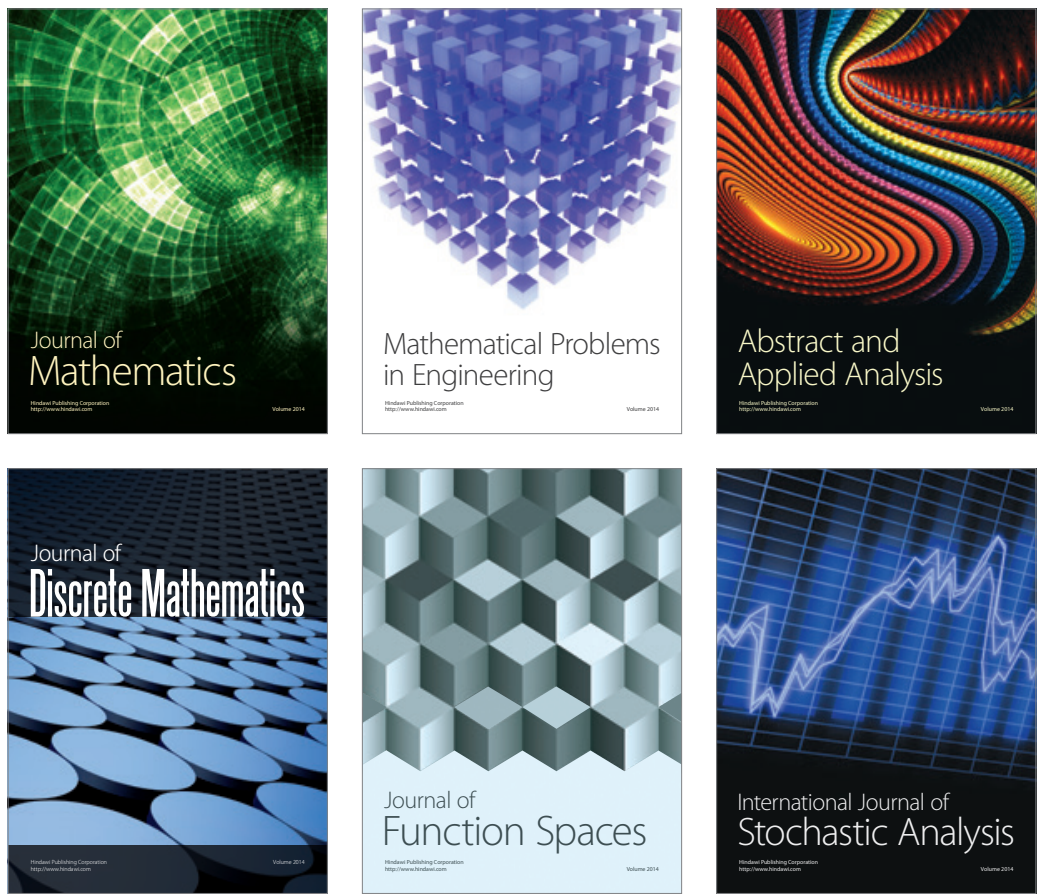

Journal of

Function Spaces

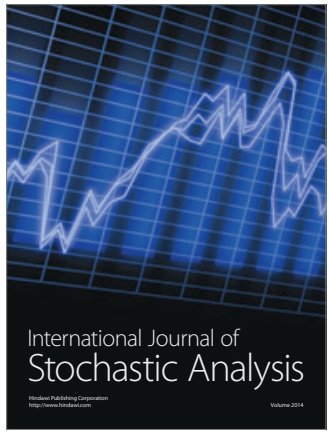

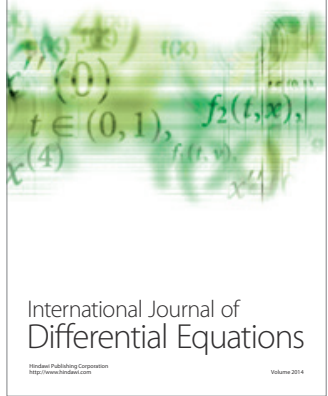
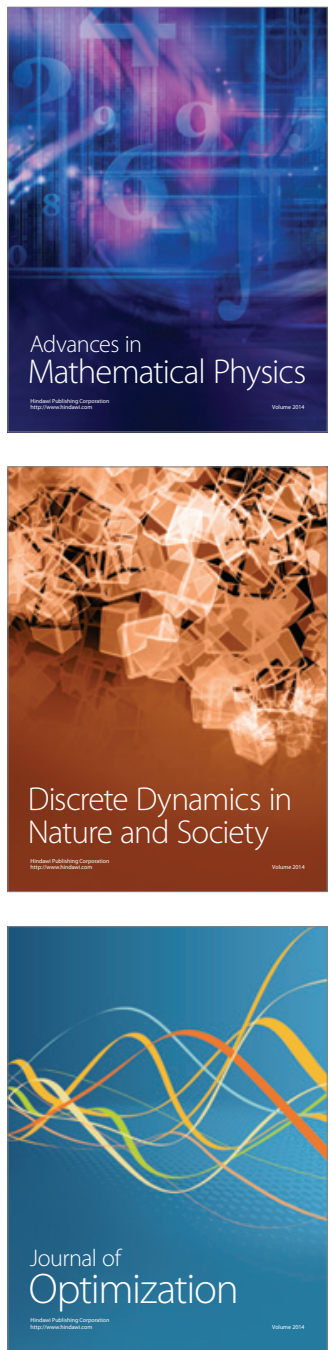